Service social

\title{
Facteurs influençant la constitution de liens entre compatriotes immigrants issus de pays ayant connu de violents conflits internes
}

\author{
Stéphanie Arsenault et Anaïs Nadeau-Cossette
}

Volume 59, numéro 2, 2013

URI : https://id.erudit.org/iderudit/1019106ar

DOI : https://doi.org/10.7202/1019106ar

Aller au sommaire du numéro

Éditeur(s)

École de service social de l’Université Laval

ISSN

1708-1734 (numérique)

Découvrir la revue

Citer cet article

Arsenault, S. \& Nadeau-Cossette, A. (2013). Facteurs influençant la constitution de liens entre compatriotes immigrants issus de pays ayant connu de violents conflits internes. Service social, 59(2), 1-15. https://doi.org/10.7202/1019106ar
Résumé de l'article

Cet article présente un état des lieux des facteurs influençant la formation de liens entre compatriotes immigrants et réfugiés issus de pays en conflits internes. Nous y évoquons les allégeances politiques et idéologiques, les identités claniques, ethniques ou religieuses, le fait même de se trouver en exil, l'existence de préjugés ou de stéréotypes à l'endroit des immigrants, la taille et la concentration territoriale de la population immigrante concernée ainsi que d'autres facteurs d'ordre personnel comme l'âge des immigrants, leur niveau de scolarité, leur origine urbaine ou rurale, leur situation économique et leur classe sociale ainsi que leur statut migratoire. L'influence de plusieurs de ces facteurs interreliés est généralement en cause et la majorité de ceux-ci ont une influence potentiellement multidirectionnelle. Nous avons cependant constaté des résultats de nature contradictoire, ce qui suggère la nécessité de poursuivre et de raffiner l'étude de ces phénomènes. 


\title{
Facteurs influençant la constitution de liens entre compatriotes immigrants issus de pays ayant connu de violents conflits internes
}

\author{
Stéphanie Arsenault \\ Anaïs Nadeau-Cossette
}

\section{RÉSUMÉ}

Cet article présente un état des lieux des facteurs influençant la formation de liens entre compatriotes immigrants et réfugiés issus de pays en conflits internes. Nous y évoquons les allégeances politiques et idéologiques, les identités claniques, ethniques ou religieuses, le fait même de se trouver en exil, l'existence de préjugés ou de stéréotypes à l'endroit des immigrants, la taille et la concentration territoriale de la population immigrante concernée ainsi que d'autres facteurs d'ordre personnel comme l'âge des immigrants, leur niveau de scolarité, leur origine urbaine ou rurale, leur situation économique et leur classe sociale ainsi que leur statut migratoire. L'influence de plusieurs de ces facteurs interreliés est généralement en cause et la majorité de ceux-ci ont une influence potentiellement multidirectionnelle. Nous avons cependant constaté des résultats de nature contradictoire, ce qui suggère la nécessité de poursuivre et de raffiner l'étude de ces phénomènes.

Mots clés : Immigration, réfugié, relations ethniques, intégration.

\begin{abstract}
This paper presents the factors influencing the creation of bonds between immigrant and refugee compatriots coming from countries in conflict. We discuss political and ideological allegiances, clannish, ethnic or religious identities, the fact of being in exile, the existence of prejudice or stereotypes against immigrants, the size and territorial concentration of the immigrant population from a specific country as well as other personal factors, such as age, education level, urban or rural origin, economic conditions, social class and, finally, migratory status. Generally speaking, we observe the inter-influence of several factors and suggest that the majority of these factors has a potentially multidirectional influence. We also note some contradictory results which suggest the need for continuing and refining future studies in this area.
\end{abstract}


L'article qui suit s'attarde aux facteurs pouvant influencer la constitution de liens entre immigrants compatriotes issus de pays en conflit. II est le fruit d'une recension des écrits scientifiques réalisée dans le cadre d'une recherche portant sur les relations entre compatriotes immigrants et réfugiés issus de pays connaissant ou ayant connu des conflits internes. Cette recherche financée par le Fonds québécois de recherche sur la société et la culture (FQRSC) prendra fin en 2013 et apportera un éclairage nouveau sur le sujet à partir de l'analyse du vécu d'immigrants d'origine bosnienne, colombienne et congolaise (RDC) établis dans la région de Québec. À la lumière des écrits scientifiques répertoriés, le nombre d'études portant spécifiquement sur ces relations dans un contexte post-migratoire paraît somme toute limité, mais certaines études ont abordé le sujet par l'analyse de thèmes connexes. Les études consultées ont analysé la réalité d'immigrants et de réfugiés provenant de Bosnie-Herzégovine, de Colombie, du Chili, de Cuba, d'Éthiopie, du Guatemala, d'Irak, du Liban, de Palestine, du Salvador, de Somalie et du Soudan, établis en Australie, au Canada, aux États-Unis et dans différents pays d'Europe et d'Afrique. Ces pays de provenance ont connu des conflits internes de nature politique, ethnique, clanique ou religieuse, où souvent plusieurs de ces dimensions se trouvaient interreliées. Les auteurs des textes recensés ont identifié de nombreux facteurs impliqués dans la création ou dans l'absence de liens entre compatriotes issus de ces pays, ou encore ont décrit la nature et la portée des liens existant entre les compatriotes étudiés. Le texte qui suit présente donc chacun des facteurs pouvant influencer favorablement ou non la création de liens au sein des différents groupes de compatriotes étudiés.

\section{LA CONSTITUTION ET LA NATURE DES LIENS ENTRE COMPATRIOTES IMMIGRANTS}

II s'avère d'abord indéniable que des immigrants provenant de pays ayant connu un conflit interne comptent sur des liens avec des compatriotes leur servant de support social, d'aide à l'installation ou de source importante d'activités socioculturelles ou politiques (Barnes et Aguilar, 2007; Bermudez, 2011; Bolzman, 2002; Change Institute-Communities and Local Government [CICLG], 2009; Doraï, 2003; Kleist, 2008; Lewis, 2010; McMicheal et Manderson, 2004; Remington Lucken, 2010; Schweitzer, Greenslade et Kagee, 2007; Wahlbeck, 1998). Des activités à caractère social comme la musique ou la danse organisées par les membres de groupes d'immigrants contribuent, on l'a reconnu, au développement d'un sentiment identitaire et d'appartenance au groupe ou à la communauté, particulièrement chez les réfugiés (Lewis, 2010). Les fêtes, soirées musicales et autres rassemblements de compatriotes offrent parfois pour certains l'occasion de créer des liens sociaux, d'échanger des nouvelles du pays d'origine, de fournir de l'information ou du support (CICLG, 2009; Schweitzer, Greenslade et Kagee, 2007; Kleist, 2008). Pour y arriver, plusieurs auteurs soulignent l'importance de compter sur des lieux rassembleurs pour les groupes, comme des mosquées, des restaurants ou des bars notamment (CICLG, 2009; Remington Lucken, 2010).

Ainsi, les membres d'un groupe de ressortissants de même origine sont reconnus comme une source potentielle de support dans le processus d'insertion des nouveaux arrivants en exil. En ce sens, pour Barnes et Aguilar (2007) et Doraï (2003), les compatriotes déjà établis au pays d'accueil peuvent servir de support émotionnel et matériel permettant de trouver un emploi, un logement ou aidant à l'apprentissage de la langue d'accueil. Malgré ces bienfaits potentiels reconnus aux relations entre compatriotes exilés, d'autres auteurs avancent que l'un 
des plus grands défis auxquels ont à faire face les réfugiés pourrait bien être celui de réinventer un sens de communauté dans leur ville d'accueil (Eastmond, 1998; Remington Lucken, 2010). La perte de ce sens de la communauté représenterait une expérience particulièrement difficile pour les réfugiés ayant vécu dans un contexte de violence organisée (Blackwell, 1993). II apparaît que les diverses différences existant entre compatriotes peuvent s'avérer tout aussi difficiles à vivre que celles présentes entre ces immigrants et les autres membres de la société d'accueil. En outre, le fait que ces immigrants soient perçus comme un tout homogène dans le pays d'accueil, alors qu'il n'en est rien, peut être une source importante de mécontentement (Mahmoud, 2011). Les notions de «communauté »- définie tantôt «par le sentiment d'appartenir et de faire partie d'un réseau de support et de relations sur lequel on peut s'appuyer » (Sarason, 1974, citée dans Barnes et Aguilar, 2007) ou encore « comme une forme de relations caractérisées par un haut degré d'intimité, une profondeur émotionnelle, un engagement moral, une cohésion sociale ainsi qu'une continuité dans le temps » (Nisbet, 1967, cité dans Kelly, 2003) - et de «communauté ethnique » se trouvent ainsi souvent utilisées de façon plus ou moins adéquate en terre d'accueil, alors que l'on se trouve parfois face à l'absence de liens sociaux solidaires ou d'amitié entre compatriotes identifiés à une même communauté. Pour nommer cette réalité, Kelly (2003) s'est intéressée à la notion de contingent community, dans un contexte où les associations dites communautaires ne reflètent pas les besoins d'une communauté, mais plutôt ceux d'un groupe construit artificiellement en réponse à une politique sociale basée sur la présomption que les immigrants issus d'un même pays forment une communauté unie. La section suivante fera donc état de divers éléments à même d'influer, dans un sens ou dans l'autre, sur les dynamiques entre compatriotes immigrants issus de pays en conflit.

\section{Les allégeances politiques et idéologiques}

Les allégeances politiques et idéologiques peuvent constituer le socle de différences importantes entre ressortissants d'un même pays origine. Celles-ci semblent parfois pouvoir constituer une force mobilisatrice importante, plus encore que la religion ou la parenté (Bermudez, 2011; Bolzman, 2002; Wahlbeck, 1998). La recherche de Wahlbeck (1998) démontre en effet que, même si les Kurdes apparaissent politiquement divisés, une force importante unit les réfugiés partageant les mêmes postures idéologiques et un passé similaire dans le pays d'origine. Ainsi, les réfugiés kurdes établis à Londres utilisent les associations politiques comme ressources facilitant leur établissement, ce qui laisse par ailleurs dans la marge les groupes sociaux n'étant pas politiquement organisés de même que les individus non politisés ou qui en ont contre la politique. Les ressortissants colombiens (Bermudez, 2011) et chiliens (Bolzman, 2002) établis en Europe ont également présenté des réalités similaires.

Par ailleurs, si, sous un premier angle d'analyse, des affiliations idéologiques ou politiques divergentes peuvent constituer des piliers facilitant la mobilisation de sous-groupes au sein d'une population provenant d'un même pays, elles peuvent, sous un second angle, manifester leur caractère limitatif à la mobilisation. En effet, dans son étude sur les réfugiés soudanais, Moro (2004) explique que les conflits violents entre les différents groupes ethniques du Soudan établis en Ouganda et en Égypte soulignent la nature politique des relations à l'intérieur de la population de réfugiés en exil. Pour Guarnizo, Sanchez et Roach (1999), dans un contexte où la 
population colombienne aux États-Unis est, de façon générale, peu impliquée politiquement, la trop grande diversité des opinions quant à la situation sociopolitique au pays d'origine divise la population, alors qu'il y a perpétuation des divisions idéologiques dans le pays d'accueil.

\section{Les identités claniques, ethniques, nationales ou religieuses}

Les distinctions ou divisions claniques, ethniques, nationales ou religieuses constituent un autre élément pouvant intervenir dans la constitution de liens entre compatriotes immigrants issus de pays en conflit. Les clans présents au sein de populations de certaines origines semblent devenir un lieu important de support, de solidarité et de sécurité pour certains nouveaux arrivants (CICLG, 2009; Kleist, 2008). Les réfugiés somaliens vivant en Angleterre, en Australie et en Nouvelle-Zélande qui comptent souvent sur les réseaux ou organisations claniques pour faciliter leur intégration en sont un bon exemple (CICLG, 2009; Schweitzer, Greenslade et Kagee, 2007).

Par ailleurs, le système de clans présent dans certaines populations d'immigrants ainsi que l'appartenance ethnique peuvent également constituer des motifs à l'origine de l'effritement des relations sociales entre compatriotes (Bermudez, 2011; Bouvier, 2007; Hopkins, 2010; Kleist, 2008; Sorenson, 1990). En guise d'exemple, le système de clans qui constitue une partie inhérente de la vie sociale somalienne a été d'une importance capitale pendant le conflit en influant sur les rapports entre les différents groupes, influence qui se poursuit en exil au sein des organisations de migrants somaliens. Ainsi, bien que ces clans constituent une source d'aide pour des réfugiés somaliens, certains organismes contribuent à amplifier la division concernée par le conflit (Hopkins, 2010; Kleist, 2008). Même si ces organisations se présentent pour la plupart comme simplement somaliennes et que peu d'entre elles affichent ouvertement une affiliation régionale, clanique ou ethnique, la majorité possède en réalité, ou se voit étiqueter, une affiliation de clan, en raison de l'origine de ses dirigeants. Dans ce contexte, les participantes à l'étude de Hopkins (2006) rapportaient beaucoup de méfiance et de réticence à fréquenter ces organisations. Qui plus est, si les clans influencent l'octroi des services à la population somalienne aux États-Unis, ils ont aussi un impact sur la quantité des organisations. Alors que la division entre les clans se reflète dans la coexistance de nombreuses petites organisations, il en résulte une diminution du financement ou des ressources disponibles pour chaque organisation. Ainsi, les services paraissent fragmentés pour les utilisateurs.

En regard de l'identité ethnique, Eastmond (2010) affirme que les liens interethniques développés après le conflit en Bosnie-Herzégovine sont généralement peu profonds, mais que de réelles amitiés interethniques ou intergroupes se créent néanmoins. C'est également le cas dans la population d'origine bosnienne établie à Boston qui semble unie malgré le conflit (Remington Lucken, 2010). Remington Lucken (2010) a pu observer que le degré de cohésion et de solidarité interethnique entre ressortissants d'un même pays différait selon les villes d'établissement. Elle conclut qu'un groupe fortement hétérogène sur le plan ethnique et religieux, peu pratiquant et s'identifiant également peu aux repères ethniques, paraît plus uni, alors qu'un groupe multiethnique comptant une majorité plus homogène, religieusement pratiquante et fortement identifiée aux repères ethniques, paraît moins ouvert à la cohésion interethnique. Mais des facteurs comme l'intensité du conflit, sa durée, le temps écoulé depuis 
la fin du conflit pourront également jouer. Un sentiment de supériorité des uns envers les autres peut également s'installer, comme c'est le cas chez les Colombiens de descendance espagnole envers les Afros-Colombiens et les Autochtones (Bermudez, 2011; Bouvier, 2007). Mahmoud (2011) rappelle que, malgré le fait que des groupes ont cohabité de façon pacifique dans le passé, à l'apparition d'un conflit, les individus tendent souvent à se rabattre sur leurs origines spécifiques pour faire face à la menace. En appui à cette constatation, Mahmoud va même, dans son étude de la situation des réfugiés soudanais au Caire, jusqu'à utiliser l'aphorisme Conflict defines origins («C'est le conflit qui définit les origines ») (2011, p. 263, 272).

II semble de plus que certaines actions des organismes officiels d'accueil puissent aussi avoir un impact sur les dynamiques de repli identitaire des réfugiés. Ce fut notamment le cas au Caire alors qu'une décision politique du Haut-Commissariat des Nations unies pour les réfugiés (HCR) eut pour effet de faire ressurgir les conflits entre deux différents groupes de réfugiés soudanais en privilégiant l'un d'entre eux au détriment de l'autre (Mahmoud, 2011).

Certains auteurs mentionnent également le peu d'intérêts communs entre compatriotes comme cause potentielle de divisions au sein des populations de réfugiés. Les études de réfugiés établis en Suède (Eastmond, 1998) et au Royaume-Uni (Williams, 2006) relèvent le fait que ces derniers considèrent souvent avoir peu en commun avec leurs compatriotes, hormis le fait de provenir d'un même pays ou d'une même nation, ce qui les incitait peu à se côtoyer.

\section{L'influence de l'exil}

Le fait d'être installé en exil constituerait un autre facteur influant sur les relations sociales entre compatriotes. Alors que, pour certains, l'exil serait bénéfique sur ce plan et contribuerait à calmer les tensions, pour d'autres il deviendrait plutôt une difficulté supplémentaire avec laquelle composer. Dans son étude sur la diaspora colombienne, Bermudez (2011) suggère en effet que, dans certains cas, les différences et les divisions au sein d'une communauté en conflit peuvent s'amenuiser en exil, permettant un certain rapprochement. Ainsi, malgré le fait que les groupes d'immigrants colombiens reproduisent habituellement les relations sociales conflictuelles du pays d'origine dans le pays d'accueil, le fait d'être retirés du climat de violence quotidienne et de tensions du pays d'origine offre de nouvelles possibilités de collaboration que certains utilisent (Bouvier, 2007). En outre, Remington Lucken (2010) mentionne le désir qu'éprouvent certains migrants de laisser le passé derrière soi afin de retrouver une vie normale, désir qui contribuerait à l'établissement de relations posées entre compatriotes. Par exemple, la solidarité interethnique observée chez les Bosniens de différentes confessions établis à Boston pourrait être le résultat d'une tolérance générée par la peur de voir le conflit ressurgir dans un contexte où les gens ne veulent plus d'ennuis et souhaitent un retour à la tranquillité. Par ailleurs, pour d'autres, ce désir de laisser le passé derrière soi se concrétiserait plutôt par l'évitement des compatriotes (Bouvier, 2007).

De façon diamétralement opposée, Blackwell (1993) estime quant à lui que les conséquences des conflits sur les individus et leurs réseaux sociaux - comme la rupture des liens sociaux, la méfiance, la peur et la perte du sens de la communauté - sont exacerbées par l'exil, ce qui rendrait les relations avec les compatriotes d'autant plus difficiles. En effet, bien 
que les réfugiés puissent entrer en contact avec des connaissances de leur pays, ou même de leur ville d'origine, le conflit au pays teinte encore les interactions sociales en exil où la peur, la méfiance et l'anxiété générées par la violence organisée au pays continuent de se manifester. La méfiance est un élément qui revient particulièrement souvent concernant les immigrants et réfugiés colombiens. Selon plusieurs études (Arsenault, 2006; Bermudez, 2011; Bouvier, 2007; Charland, 2006; Guarnizo et al., 1999), le conflit colombien aurait causé un sentiment très répandu de peur et de manque de confiance entre compatriotes établis à l'étranger. Si les réfugiés se fréquentent à l'occasion, peu d'entre eux se disent très proches et certains restent volontairement distants de leurs compatriotes. De plus, pour ces mêmes raisons, des réfugiés colombiens éviteraient de fréquenter certains organismes d'accueil au Québec: «La faible fréquentation des organismes d'accueil et d'établissement par les réfugiés colombiens trouve ainsi son origine dans l'effritement des relations de confiance sociale que la structure d'accueil dans son ensemble contribue à reproduire » (Charland, 2006, p. 125). D'autres auteurs rapportent quant à eux une certaine confusion que peuvent ressentir les immigrants envers leurs compatriotes, à savoir si ces derniers sont des «amis » ou des « ennemis », une confusion qu'alimente le développement d'un sentiment de méfiance (Blackwell, 1993; Mahmoud, 2011).

II semble en outre que des relations intracommunautaires peu harmonieuses peuvent mener à l'exclusion de certains compatriotes qui vivront de l'isolement social. McMichael et Manderson (2004), dans leur recherche sur les Somaliennes établies en Australie, parlent de la rupture des réseaux sociaux engendrée par le conflit dans le pays d'origine. Ainsi, la tristesse, l'anxiété et la solitude sont fréquemment attribuées à la dissolution des liens qu'elles entretenaient auparavant avec les autres Somaliens.

Cependant, pour certains immigrants issus de pays en conflit, l'isolement de leurs pairs est un choix, un mécanisme de défense visant à échapper à l'anxiété et aux souvenirs douloureux qui refont surface lors d'interactions sociales avec des compatriotes (Blackwell, 1993; Bouvier, 2007; Charland, 2006; Miller et al., 2002a; Miller et al., 2002b). Charland (2006) observe de tels comportements chez les réfugiés colombiens au Québec. II semble qu'étant donné la méfiance très présente dans ce groupe de compatriotes, les relations interpersonnelles de confiance soient moins importantes et que le repli sur soi et l'isolement deviennent des stratégies de défense. Ainsi, l'isolement social devient une protection contre cette source importante de stress que représente la rencontre de compatriotes (Miller et al., 2002a ; Miller et al., 2002b).

En outre, d'autres chercheurs ont relevé que certains immigrants n'évitaient pas leurs compatriotes de façon générale, mais qu'ils évitaient plus spécifiquement les rassemblements sociaux ou culturels de leur communauté (Bermudez, 2011; Lewis, 2010). Les fêtes communautaires peuvent, comme nous l'avons souligné plus tôt, être un lieu de solidarité et de création de liens sociaux utiles, mais elles peuvent aussi en effet être perçues négativement comme des enclaves traditionnelles faisant la promotion d'un isolement culturel, où des épisodes de violence surviennent à l'occasion (Bermudez, 2011; Lewis, 2010) et où circulent beaucoup de commérages, ce à quoi certains ne souhaitent guère participer (Remington Lucken, 2010). 
Le vécu personnel de la guerre, l'exposition directe au conflit et les traumatismes qui lui sont liés sont des expériences particulièrement importantes qui influent sur le degré de confiance possible entre compatriotes, les relations intracommunautaires et le pardon (Blackwell, 1993; Kelly, 2003; McMichael et Manderson 2004; O'Loughlin, 2010; Sorenson, 1990). Selon Blackwell (1993), le vécu lié à l'exil et à la violence organisée peut causer un traumatisme ayant des conséquences non seulement sur la personne, mais également sur sa famille, sur son réseau social et sur ses dynamiques relationnelles avec ses compatriotes. L'étude de McMichael et Manderson (2004), s'intéressant aux femmes somaliennes établies en Australie, illustre les conséquences de la guerre sur les relations au sein de cette population réfugiée. Alors que la solidarité, le partage, la réciprocité, la confiance et le soutien occupaient une place centrale dans leurs souvenirs de la vie dans le pays d'origine, la situation des relations sociales s'avère toute autre en terre d'accueil. Les femmes interrogées considèrent la détérioration de la cohésion sociale de la communauté somalienne, l'ébranlement des relations sociales, la diminution de la confiance ainsi que l'effritement des réseaux de solidarité comme le résultat du déplacement et de la guerre. La recherche de Kelly (2003) soulève les mêmes constatations avec les réfugiés bosniaques établis en Grande-Bretagne. Ceux-ci affirment que leurs relations avec leurs amis d'avant la guerre sont maintenant sévèrement perturbées ou totalement détruites. En plus d'ébranler les réseaux sociaux, la guerre a donc affecté leur reconstruction au pays d'accueil puisque les compatriotes se font désormais plus difficilement confiance.

\section{Les stéréotypes}

Les stéréotypes ou les préjugés dont sont affublés certains immigrants ou réfugiés dans le pays d'accueil ont aussi un rôle à jouer (Bermudez, 2011; Bouvier, 2007; Guarnizo et al., 1999). Selon Guarnizo et ses collaborateurs (1999), le fait que la population colombienne établie aux États-Unis soit peu unie est directement lié à l'image de la Colombie et aux stéréotypes qui lui sont associés, notamment concernant le trafic de la drogue. Ce préjugé largement répandu incite les immigrants colombiens à éviter leurs compatriotes pour garder leur propre réputation intacte. De la même façon, l'étude de Bermudez (2011) a laissé voir que les ressortissants colombiens démontraient beaucoup de réserves à être associés à leurs compatriotes affublés de stéréotypes négatifs ou qui propageaient des images critiques ou négatives de leur pays d'origine en terre d'accueil, deux réalités déplaisant à de nombreux répondants.

\section{L'influence du milieu urbain}

Certains autres facteurs sont également à relever. La taille de la population de réfugiés dans un lieu donné en est un (Guarnizo et al., 1999; Remington Lucken, 2010; Sorenson, 1990), mais le sens de l'influence exercée par celle-ci ne fait pas consensus. Remington Lucken (2010) suggère qu'une population importante d'immigrants serait moins unie qu'une peu nombreuse puisque, dans ce cas, les réfugiés tendraient à tisser des liens étroits plutôt qu'à se diviser en sous-groupes. À l'opposé, Guarnizo et ses collaborateurs (1999), ayant étudié deux communautés colombiennes établies dans des villes différentes des États-Unis, avancent quant à eux qu'une population moins importante fournit moins de possibilités de développer des contacts avec des compatriotes et donc ne contribuent pas à renforcer les liens intracommunautaires. À ce sujet, Eastmond (1998) précise que les contrastes entre les 
idéologies et les pratiques sociales des réfugiés associés à une origine en particulier sont davantage évidents dans une petite population ayant un nombre limité de membres et freineront donc les rassemblements entre personnes qui s'identifient comme différentes, voire opposées. Selon cette logique, une population réfugiée plus nombreuse en milieu urbain faciliterait les interactions puisqu'elle aurait accès à davantage de connaissances antérieures qui présentent les mêmes affinités sociales. D'autres études ont soulevé le caractère ambigu de l'influence de la taille d'une population réfugiée sur son potentiel relationnel: alors que pour certains une communauté plus nombreuse offre un grand capital social, pour d'autres ayant quitté leur pays pour des raisons de sécurité, elle devient une source de danger (Williams, 2006).

Certaines caractéristiques de la ville d'accueil influencent également les liens entre compatriotes immigrants, puisque ces derniers varient selon les endroits d'établissement étudiés (Bouvier, 2007). Remington Lucken (2010) observe que les villes dans lesquelles des vagues précédentes d'immigrants s'étaient établies et avaient formé des communautés basées sur des identités ethno-religieuses comprenaient maintenant des quartiers et des institutions ethniques dans lesquelles s'intégraient les nouveaux venus, perpétuant ainsi une forme de ségrégation, comme ce fut le cas à Chicago. Une ville comme Boston, n'ayant pas connu d'immigration en provenance d'ex-Yougoslavie avant le conflit, a plutôt reçu les réfugiés ensemble, indistinctement de leur appartenance ethnique, ce qui aurait favorisé leur cohésion et renforcé leur sentiment d'appartenance commune.

La concentration territoriale dans les villes influe également sur les relations entre compatriotes (Guarnizo et al., 1999; Remington Lucken, 2010; McMichael et Manderson, 2004). En effet, dans le cas de la population d'origine bosnienne établie à Boston (Remington Lucken, 2010), la proximité géographique de leurs habitations dans les premières années de leur installation facilitait leurs interactions alors que les déménagements couplés avec le temps à une dispersion géographique grandissante ont fait des rencontres et des rassemblements de la communauté des événements plus rares. Cela pousse l'auteure à affirmer qu'une plus grande concentration territoriale peut faciliter les liens intracommunautaires, ce qui a aussi été observé chez les Colombiens établis à Los Angeles (Guarnizo et al., 1999).

\section{Le rôle des leaders}

Par contre, la concentration territoriale comporterait aussi des inconvénients en regard des relations entre compatriotes (Colic-Peisker et Walker, 2003; Schweitzer, Greenslade et Kagee, 2007). En effet, la compétition et la pression exercées par les compatriotes concernant un établissement réussi et un bon statut social, symbolisés principalement par l'habitation d'une confortable maison familiale, semblent plus importantes pour les personnes vivant dans un secteur à forte concentration ethnique. Ainsi, ce type de milieu permet souvent un haut degré de soutien de la part de la communauté, mais aussi un fort degré de contrôle et de pression provenant des compatriotes.

L'influence des leaders des communautés sur l'évolution des relations entre compatriotes immigrants issus de pays en conflits est aussi à considérer. Ceux-ci joueraient un rôle significatif dans les efforts menant à minimiser les différences entre les sous-groupes, à 
encourager les valeurs communes et à rappeler la cohabitation agréable d'avant-guerre, ce qui contribue à la cohésion d'une communauté (Remington Lucken, 2010). Pour Mahmoud (2011), la formation de leaders communautaires positifs au sein des communautés de réfugiés pouvant faire la promotion de la paix plutôt qu'encourager les divisions constitue d'ailleurs une piste de solution à explorer permettant de ne pas perpétuer en exil les conflits du pays d'origine.

\section{Facteurs d'ordre personnel}

Dans la littérature, plusieurs caractéristiques propres aux immigrants semblaient elles aussi avoir un impact sur leurs relations avec leurs compatriotes. En effet, l'âge, le niveau de scolarisation, la situation économique, la classe sociale, le milieu d'origine (urbain/rural) et le statut d'immigration sont autant de facteurs pouvant avoir une influence sur les relations sociales des immigrants avec leurs compatriotes. Selon les études menées par O'Loughlin (2010) en Bosnie et par le CICLG (2009) au Royaume-Uni, les jeunes sont plus à risque de vivre des conflits de cette nature, alors que les plus âgés semblent plus ouverts ou plus tolérants aux relations interethniques. Dans le cas de la Bosnie-Herzégovine, l'auteur émet l'hypothèse que les personnes plus âgées ont connu, avant le conflit, une Yougoslavie tolérante et unie, où la cohabitation interethnique ne posait pas de problème et que pour cette raison ils pourraient, après le conflit, maintenir des liens interethniques, à l'instar du passé. Au RoyaumeUni, le système de clans somalien, qui joue un rôle politique, social et identitaire important, continue encore aujourd'hui d'avoir un impact sur la communauté, et ce, même en exil. Les résultats de l'étude du CICLG (2009) démontrent que les aînés tendent à jouer un rôle pacificateur lorsque surviennent des conflits de ce type alors que, chez les jeunes, le système de clans mène à des divisions importantes, souvent source de conflits.

Le statut d'immigration constitue un autre facteur autour duquel les relations sociales des compatriotes immigrants et réfugiés peuvent se définir. Le statut de réfugié semble souvent faire l'objet de litige au sein même des groupes d'immigrants et de réfugiés originaires de pays en conflits, alors que l'on y construit des catégorisations de « vrais » et de « faux » réfugiés, ou encore de réfugiés qui « méritent » leur statut et de ceux qui auraient « profité » du système en place, mais dont la vie n'aurait pas été réellement en danger (Lewis, 2010). Dans ces cas, les immigrants se distinguent entre eux en fonction des raisons supposées les ayant poussés à quitter leur pays d'origine et des moyens pris pour y arriver.

L'influence du statut peut aussi être observée sous un autre angle. Toujours selon Lewis (2010), les immigrants dont le statut est précaire ou ceux nouvellement arrivés vont participer davantage aux rassemblements communautaires car ces derniers procureraient un sentiment de sécurité et d'appartenance dans le pays d'accueil. Bouvier (2007) est de l'avis contraire. Elle suggère plutôt que les immigrants dont le statut est incertain tendent à éviter de côtoyer leurs compatriotes afin de passer inaperçus, ou encore parce qu'ils préfèrent ne pas tisser de liens, étant donné un retour possible dans leur pays d'origine. Williams (2006) suggère finalement que les réfugiés ayant été rejetés de leur communauté d'origine tisseront peu de liens avec leurs compatriotes au pays d'accueil. Dans le même sens, Bermudez (2011) suggère que les immigrants ayant un statut de réfugiés vivraient plus de méfiance et de peur envers leurs compatriotes que les autres catégories d'immigrants. 
Une autre source potentielle de divisions, mentionnée de façon importante dans la littérature, est la région ou la ville d'origine au pays. Alors que certains auteurs abordent le sujet selon la région spécifique de provenance, d'autres parlent plutôt d'origine urbaine ou rurale au sens large. Selon plusieurs auteurs, la fragmentation sociale observée chez certains groupes de migrants, notamment chez les Colombiens aux États-Unis, au Royaume-Uni et en Espagne, se fait selon des lignes de faille séparant les régions (Bermudez, 2011; Bouvier, 2007; Guarnizo et al., 1999). Bien que Guarnizo et ses collaborateurs (1999) suggèrent d'approfondir les recherches concernant les effets du régionalisme, ils peuvent néanmoins affirmer que certains migrants colombiens aux États-Unis limitent leurs interactions personnelles et professionnelles aux individus de leur région d'origine et que des divisions à ce sujet sont observées au sein des associations socioculturelles de cette communauté. Sorenson (1990) mentionne que, pour la population érythréenne, l'origine régionale au pays a beaucoup d'importance. En effet, il semble que cette différence ait un impact sur la cohésion de la communauté, empêchant le renforcement d'une identité érythréenne commune. Cependant, l'auteur souligne que, même si ces affiliations régionales ont contribué au conflit dans le passé, la communauté tente aujourd'hui de leur accorder moins d'importance en prohibant l'évocation du sujet, le rendant pour ainsi dire tabou. La situation de la communauté somalienne de Londres paraît semblable et connaît aussi des divisions en fonction de la région d'origine au pays (CICLG, 2009).

Tel que mentionné précédemment, en plus des tensions intracommunautaires liées à la région d'origine, certains auteurs observent également un clivage entre les immigrants originaires de milieux urbains et ceux originaires de milieux ruraux (Hopkins, 2010). Dans l'étude de Lewis, certains répondants affirment ne pas vouloir participer aux rassemblements de la communauté kurde, car ils se sentent différents des autres compatriotes y participant qui sont just country people (2010, p. 578). Selon Remington Lucken, il semble même que l'origine rural ou urbaine soit parfois plus importante que l'origine ethnique: «According to refugee resettlement officials, the rural/urban divide can create a greater gulf than ethnic affiliation » («Selon les responsables de l'installation des réfugiés, la distinction rural/urbain peut créer une division plus grande que l'affiliation ethnique ») (2010, p. 197). Selon certains auteurs, les individus provenant de milieux ruraux vivaient souvent de façon plus isolée au pays, avaient moins de contacts avec les autres groupes et accordaient davantage d'importance à leur origine ethnique et religieuse dans la définition de leur identité (Mahmoud, 2011; Remington Lucken, 2010). Mahmoud (2011) observe également, dans le cadre de son étude des réfugiés soudanais établis au Caire, que cette différence au sein de la communauté est marquante, et parmi les plus cruciales. En effet, les réfugiés originaires de milieux urbains et les gens plus scolarisés semblent moins attachés à leurs tribus ou groupes d'appartenance et aux traditions qui y sont rattachées, ce qui, dans un contexte de conflit ethnico-religieux, peut avoir une incidence certaine sur les relations entre gens issus d'un même pays. En outre, comme nous venons de le voir, le niveau de scolarisation semble souvent imbriqué avec l'origine rurale ou urbaine des compatriotes, les deux éléments se trouvant fréquemment évoqués conjointement par les auteurs, en relation l'un avec l'autre, comme motifs menant à éviter de côtoyer certains compatriotes (Hopkins, 2010; Lewis, 2010; Remington Lucken, 2010). 
La situation économique des nouveaux arrivants et celle de ceux préalablement établis peut aussi influer sur les relations qu'ils entretiennent avec les membres de leur famille et avec leurs compatriotes. Les répondantes somaliennes de l'étude de McMichael et Manderson (2004) suggèrent que la pauvreté a mené à la diminution de la réciprocité et de la redistribution informelle de ressources entre compatriotes, ce qui a eu comme conséquence d'éroder les réseaux sociaux. Plusieurs de ces femmes versent par ailleurs de l'argent à leurs familles et à leurs proches restés au pays, ce qui nuit à leur capacité d'assister financièrement leurs compatriotes demeurant dans la même ville d'accueil. Les contraintes financières diminueraient ainsi les habiletés des femmes à maintenir des liens sociaux et un sens de la communauté. Du côté des immigrants salvadoriens établis aux États-Unis, le manque de ressources financières dans les organisations communautaires de services et l'absence de participation des réfugiés de longue date ont contribué à créer un climat inhospitalier pour les nouveaux arrivants qui trouvent peu de soutien (Menjívar, 1997).

La classe sociale à laquelle s'identifient ou sont identifiés les immigrants constitue une autre source de fragmentation des réseaux sociaux chez les immigrants étudiés (Bermudez, 2011; Bouvier, 2007; Guarnizo et al., 1999; Hopkins, 2010; Lewis, 2010; Remington Lucken, 2010). Selon Bermudez (2011), Bouvier (2007) et Guarnizo et ses collaborateurs (1999), la division selon la classe sociale est particulièrement importante chez les Colombiens. La diaspora colombienne aux États-Unis serait en effet caractérisée par une multitude de petites communautés ayant peu en commun et les organisations se formeraient autour de regroupements d'immigrants des classes jugées supérieures. Selon ces auteurs, la présence d'un classisme profond, bien ancré, est un facteur déterminant dans l'explication du nombre peu élevé d'organisations communautaires colombiennes, de natures culturelle ou civique. Qui plus est, les résultats de l'étude de Lewis (2010) démontrent bien que les participants, incluant des Kurdes, des Iraniens, des Zimbabwéens et des Somaliens, évitaient les rassemblements de leur communauté, principalement en raison des différences de classes perçues avec leurs compatriotes. Par ailleurs, pour d'autres, l'exil offre la possibilité de développer et d'entretenir des relations sociales qui auraient été impossibles au pays d'origine puisque, dans l'étude de Bermudez (2011), des immigrants colombiens affirment avoir pu, après la migration, rencontrer et tisser des liens avec des compatriotes de toutes classes sociales, ce qui semblait impensable au pays.

\section{ConClusion}

Pour conclure, de nombreux facteurs peuvent donc intervenir dans la formation ou l'absence de formation de liens entre compatriotes immigrants issus de pays en conflits. Nous avons évoqué les allégeances politiques et idéologiques, les identités claniques, ethniques ou religieuses, le fait même de se trouver en exil, l'existence de préjugés ou de stéréotypes à l'endroit des immigrants, la taille et la concentration territoriale de la population immigrante concernée ainsi que d'autres facteurs d'ordre personnel comme l'âge des immigrants, leur niveau de scolarité, leur origine urbaine ou rurale, leur situation économique et leur classe sociale ainsi que leur statut migratoire. L'influence conjuguée de plusieurs de ces facteurs est généralement en cause. La majorité de ces facteurs ont une influence ambivalente (tantôt favorable, tantôt défavorable), dépendamment du croisement particulier des différents facteurs en cause. Nous 
nous trouvons donc face à une constellation de situations fort diversifiées et toujours complexes. Nous avons par ailleurs constaté plusieurs résultats de nature contradictoire selon les études, ce qui suggère la nécessité de poursuivre et de raffiner l'étude de ces phénomènes. À la lumière des textes répertoriés, il paraît par ailleurs évident, bien que les liens entre compatriotes possèdent un caractère positif et un pouvoir de soutien pour certains, qu'il est loin d'en être ainsi pour tous et dans toutes les circonstances. En effet, les recherches identifient clairement les limites en regard du développement de ces liens ainsi que le potentiel conflictuel ou perturbateur qu'ils comportent pour de nombreuses personnes immigrantes ou réfugiées. Ce constat nous amène à insister sur le caractère hétérogène de la population immigrante et réfugiée provenant d'un même pays et sur les barrières plus ou moins grandes que peuvent occasionner les différences qu'elle abrite en regard des liens pouvant être établis entre ressortissants de pays communs. La valorisation d'initiatives collectives, formelles ou non, émanant de l'État ou encore d'immigrants et de réfugiés d'une origine donnée et s'adressant à cette même population, ne devrait jamais éclipser l'importance de faciliter et de promouvoir également des initiatives collectives et des services à caractère multiethnique, incluant ceux destinés à l'ensemble de la population, donc non spécifiques aux immigrants, et qui permettent de tabler sur d'autres facteurs d'identification, d'appartenance et de mobilisation que le pays d'origine. Dans cette même ligne, l'étude de la place et du rôle des relations entre compatriotes comparés à ceux des relations immigrants/non-immigrants dans l'intégration des nouveaux venus méritent d'être poursuivie. La recension réalisée ne permet pas de mettre clairement en évidence ce qui distingue les immigrants et réfugiés provenant de pays en conflits des immigrants provenant plutôt de pays pacifiques sur le plan du développement de relations entre compatriotes et une recension comparative en ce sens mériterait également d'être poursuivie.

\section{Stéphanie Arsenault}

Professeure agrégée

École de service social, Université Laval Doctorat en anthropologie sociale, Université de Grenade, Espagne Maîtrise en service social, Université Laval 


\section{BIBLIOGRAPHIE}

Arsenault, S. (2006). « Transnacionalismo, el caso de los refugiados colombianos en Quebec ». Thèse de doctorat non publiée, Université de Grenade, Espagne.

Barnes, D.M. et R. Aguilar (2007). « Community Social Support for Cuban Refugees in Texas », Qualitative Health Research, vol. 17, $\mathrm{n}^{\circ}$ 2, p. 225-237.

Bermudez, A. (2011). "The "Diaspora Politics" of Colombian Migrants in the UK and Spain », International Migration, vol. 49, n 3, p. 125-143.

Blackwell, R. (1993). «Disruption and Reconstitution of Family, Network and Community System Following Torture, Organised Violence and Exile », dans J. Wilson et B. Raphael, The International Handbook of Traumatic Stress Symptom, New York, Plenum Press, p. 733741.

Bolzman, C. (2002). « De l'exil à la diaspora : l'exemple de la migration chilienne », Autrepart, $n^{0} 22$, p. 91-107.

Bouvier, M. (2007). «A Reluctant Diaspora: The Case of Colombia » dans H. Smith et P. Stares, Conflict: Peace-Makers or Peace-Wreckers, Tokyo, United Nations University Press, p. 129-152.

Change Institute, Communities and Local Government [CICLG] (2009). The Somali Muslim Community in England-Understanding Muslim Ethnic Communities, Londres, Communities and Local Government Publications.

Charland, M. (2006). « La confiance au cœur de l'exil : Récit de réfugiés colombiens ». Mémoire de maîtrise non publié, Université Laval, Canada.

Colic-Peisker, V. et I. Walker (2003). « Human Capital, Acculturation and Social Identity: Bosnian Refugees in Australia », Journal of Community and Applied Social Psychology, vol. $13, n^{0} 5$, p. 337-360.

Doraï, M. K. (2003). «Palestinian Emigration from Lebanon to Northern Europe: Refugees, Networks, and Transnational Practices », Refugee, vol. 21, n² 2, p. 23-31.

Eastmond, M. (2010). «Introduction: Reconciliation, reconstruction, and everyday life in wartorn societies », Focaal-Journal of Global and Historical Anthropology, n 57, p. 3-16.

Eastmond, M. (1998). «Nationalist Discourses and the Construction of Difference: Bosnian Muslim Refugees in Sweden », Journal of Refugee Studies, vol. 11, n² 2, p. 161-181.

Guarnizo, L.E., A.I. Sanchez et E. Roach (1999). «Mistrust, fragmented solidarity, and transnational migration: Colombians in New York City and Los Angeles », Ethnic and Racial Studies, vol. 22, $\mathrm{n}^{\circ} 2$, p. 367-396.

Hopkins, G. (2010). «A changing sense of Somaliness: Somali women in London and Toronto », Gender, Place and Culture, vol. 17, n 4, p. 519-538. 
Hopkins, G. (2006). « Somali Community Organizations in London and Toronto: Collaboration and Effectiveness », Journal of Refugee Studies, vol. 19, $n^{\circ} 3$, p. 361-380.

Kelly, L. (2003). «Bosnian Refugees in Britain: Questioning Community », Sociology, vol. 37, $\mathrm{n}^{\circ} 1$, p. 35-49.

Kleist, N. (2008). « Mobilising "The Diaspora": Somali Transnational Political Engagement », Journal of Ethnic and Migration Studies, vol. 34, $\mathrm{n}^{\circ} 2$, p. 307-323.

Lewis, H. (2010). « Community Moments : Integration and Transnationalism at Refugee Parties and Events », Journal of Refugee Studies, vol. 23, $n^{\circ}$ 4, p. 571-588.

Mahmoud, H.W. (2011). " "Conflict Defines Origin": Identity Transformations of Sudanese Refugees in Cairo », Conflict Resolution Quaterly, vol. 28, n 3, p. 263-289.

McMichael, C. et L. Manderson (2004). « Somali Women and Well-Being: Social Networks and Social Capital among Immigrant Women in Australia », Human Organization, vol. 63, $\mathrm{n}^{\circ} 1$, p. 88-99.

Menjívar, C. (1997). «Immigrant Kinship Networks and the Impact of the Receiving Context: Salvadorans in San Francisco in the Early 1990 s », Social Problems, vol. 44, n 1, p. 104123.

Miller, K.E., G.J. Worthington, J. Muzurovic, S.Tipping et A. Goldman (2002a). « Bosnian Refugees and the Stressors of Exile », American Journal of Orthopsychiatry, vol. $72, \mathrm{n}^{\circ} 3$, p. 341-354

Miller, K.E., S. Weine, A. Ramic, N. Brkic, Z. Djuric Bjedic, A. Smajkic, E. Boskailo et G. Worthington (2002b). «The Relative Contribution of War Experiences and Exile-Related Stressors to Levels of Psychological Distress Among Bosnian Refugees », Journal of Traumatic Stress, vol. 15, n 5, p. 377-387.

Moro, L.N. (2004). « Interethnic Relations in Exile: The Politics of Ethnicity among Sudanese Refugees in Uganda and Egypt », Journal of Refugee Studies, vol. 17, n 4, p. 420-436.

O'Loughlin, J. (2010). «Inter-ethnic friendships in post-war Bosnia-Herzegovina: Sociodemographic and places influences », Ethnicities, vol. 10, $n^{\circ} 1$, p. 26-54.

Remington Lucken, K. (2010). «Identity Matters: Bosnian Identity Maintenance in a PostMigration Setting ». Thèse de doctorat, Boston University, États-Unis.

Schweitzer, R., J. Greenslade et A. Kagee (2007). « Coping and resilience in refugees from the Sudan: a narrative account », Australian and New Zealand Journal of Psychiatry, vol. 41, $\mathrm{n}^{0} 3$, p. 282-288.

Sommers, M. (1995). «Representing Refugees: The Role of Elites in Burundi Refugee Society », Disaster, vol. 19, $\mathrm{n}^{\circ} 1$, p. 19-25.

Sorenson, J. (1990). «Opposition, Exile and Identity: The Eritrean Case », Journal of Refugee Studies, vol. 3, n $\mathrm{n}^{\circ}$, p. 298-319. 
Wahlbeck, Ö. (1998). «Community Work and Exile Politics: Kurdish Refugee Associations in London », Journal of Refugee Studies, vol. 11, $n^{\circ} 3$, p. 215-230.

Williams, L. (2006). «Social Networks of Refugees in the United Kingdom: Tradition, Tactics and New Community Spaces », Journal of Ethnic and Migration Studies, vol. 32, $n^{\circ}$ 5, p. 865879. 